\title{
1 Stabilization of species coexistence in spatial models through the aggregation-segregation effect 2 generated by local dispersal and nonspecific local interactions
}

\author{
3 Matteo Detto and Helene C. Muller-Landau \\ 4 Smithsonian Tropical Research Institute, Panama City, Panama \\ 5 Corresponding author: Matteo Detto \\ 6 Unit 9100, Box 0948 \\ 7 DPO AA 34002-9998, USA \\ 8 Telephone: $+507670-05002$ \\ 9 Email: DettoM@si.edu
}

11 ABSTRACT. Spatial interactions are widely acknowledged to play a significant role in sustaining diversity 12 in ecological communities. However, theoretical work on this topic has focused on how spatial processes 13 affect coexistence of species that differ in their strategies, with less attention to how spatial processes 14 matter when competitors are equivalent. Furthermore, though it is recognized that models with local 15 dispersal and local competition may sustain higher diversities of equivalent competitors than models in 16 which these are not both localized, there is debate as to whether this reflects merely equalizing effects 17 or whether there is also a stabilizing component. In this study, we explore how dispersal limitation and 18 nonspecific local competition influence the outcome of species coexistence in communities driven by 19 stochastic drift. We demonstrate that space alone acts as a stabilizing factor in a continuous space 20 model with local dispersal and competition, as individuals of rare species on average experience lower 21 total neighborhood densities, causing per capita reproductive rates to decrease systematically with 22 increasing abundance. These effects prolong time to extinction in a closed system and enhance species 23 diversity in an open system with constant immigration. Fundamentally, these stabilizing effects are 24 obtained when dispersal limitation interacts with local competition to generate fluctuations in 25 population growth rates. Thus this effect can be considered a fluctuating mechanism similar to spatial 26 or temporal storage effects, but generated purely endogenously without requiring any exogenous 27 environmental variability or species dissimilarities.

28 Keywords: spatially explicit model, nonspecific local interactions, dispersal limitation, time to extinction, 29 species diversity, relative species abundance 
In ecological communities where individuals have limited ability to move (e.g. plants and soil microbes), local spatial dynamics often play a central role in determining population and community structure and dynamics (P Chesson, 2000; Pacala, 1997; Stoll and Prati, 2001; Turnbull et al., 2007; Webb et al., 2007). The ecological importance of space has motivated the development of spatially explicit theoretical approaches (Dunning et al., 1995; Durrett and Levin, 1994; Perc et al., 2013). This includes individual-based simulation models (on a lattice or continuous space) and approximate analytical models such as moment equations (Bolker and Pacala, 1997) and reaction-diffusion equations (Britton, 1986). Spatially explicit theory has demonstrated the limitations of spatially implicit competition models and classical neutral theory; for example, spatial dynamics can alter the parameter space in which stable coexistence is possible (Benjamin $\neg$ †M. $\neg$ +Bolker et al., 2003) and lead to biased estimations and interpretations of model parameters (Etienne and Rosindell, 2011; Pacala and Levin, 1997).

To date, spatial ecological theory has generated many interesting results on how spatial processes alter niche mechanisms of coexistence, though little attention has been paid to exactly how spatial interaction terms matter when competitors are equivalent. Spatial dynamics have been investigated extensively in models of resource or apparent competition (Bonsall and Hassell, 2000; Molofsky et al., 2002; O'Brien et al., 2007; Pacala, 1987), habitat partitioning (P Chesson, 2000), competitioncolonization and other life history tradeoffs (e.g. Bolker and Pacala, 1999; Chave et al., 2002; King and Hastings, 2003), and specialized natural enemies (e.g. Adler and Muller-Landau, 2005; Sedio and Ostling, 2013). It has been recognized that even in the absence of species differences, spatial localization matters (Hanski, 1981; Slatkin, 1974); however, there is a dearth of research on how the spatial interaction terms affect population and community dynamics in these cases. Models with ecologically identical species are typically addressed only in the context of null model comparison, where the focus is on understanding spatial niche mechanisms (e.g. Sedio and Ostling, 2013).

Surprisingly, there remains a fundamental disagreement as to whether spatial effects on their own can contribute to stabilizing species coexistence, or if they are merely equalizing. A mechanism is said to be stabilizing if it increases intraspecific negative density-dependence relative to interspecific negative density dependence, thus leading a species per capita population growth rate to increase if it becomes rare (Peter Chesson, 2000). In contrast, equalizing mechanisms reduce fitness differences among species, thereby slowing eventual extinction, but provide no advantage to a species when rare nor disadvantage when abundant.

Classical niche mechanisms such as resource partitioning, life history tradeoffs, and habitat specialization are all well understood to be stabilizing. Proper understanding of the role of spatial processes alone is an important starting point for any quantification of stabilizing effects of these or other niche mechanisms in spatial models and observations. If local interactions themselves have stabilizing effects that are not considered, these may confound the stabilization provided by spatial

67 niche mechanisms.

Local dispersal and competition lead to conspecific aggregation and species segregation, and 
with heterospecifics (Holyoak and Loreau, 2006; Murrell et al., 2001). By reducing heterospecific competition, these spatial structures could slow ecological dynamics (Hurtt and Pacala, 1995), a finding that has motivated the formulation of a segregation hypothesis. This hypothesis states that "finite dispersal and spatially local interactions lead to spatial structure that enhances ecological stability (resilience) and biodiversity (Pacala, 1997)".

However, it is possible for local aggregation of a species to accelerate exclusion and reduce the parameter space where coexistence occurs (Chesson and Neuhauser, 2002; Neuhauser and Pacala, 1999). Similarly, in models of invasion, spatial structures and life-history tradeoff play a central role in determining the outcome of an invasion, but intraspecific competition intensifies so much that competitive dynamics are actually faster than in the nonspatial case (Bolker et al., 1999).

It is unclear if these mechanisms are expected to have larger effects among similar competitors, and therefore to be particularly important for spatially explicit neutral community models. Analytical approximations derived by spatial stochastic processes, which describe the average population dynamics, have shown that asymmetry in conspecific and heterospecific interaction scales is necessary to achieve stable coexistence of otherwise equal competitors (Murrell and Law, 2003).

It is important to note that the presence of conspecific aggregation and heterospecific segregation alone, which emerge in many simulations, is not a sufficient condition for stabilization. In neutral lattice models in which all cells are occupied, for example, total neighborhood densities of conspecifics plus heterospecifics remain invariant even though conspecifics are highly clustered, and in this case there can be no stabilization as all individuals experience equal local competition. In such models spatial structures could enhance coexistence by reducing potential rate of change in abundance of species, i.e. reducing the stochastic drift. This mechanism acts in a similar manner of an equalizing mechanism (Chave et al., 2002; Neuhauser and Pacala, 1999), even though the definition of equalization does not apply well here because all species are identical, so fitness differences cannot be reduced.

In continuous systems with local dispersal and competition, spatial structures continuously form and dissipate (Detto and Muller-Landau, 2016). These spatiotemporal dynamics may generate covariance between local population densities and population growth rates, for example, isolating individuals of species that are at low abundance and in danger of drifting to extinction. Such temporary spatial refugia alleviate competitive pressure and increase the probability that these species recover from rarity. Although spatial segregation is unlikely to be permanent, it could last long enough to significantly affect population dynamics, but the extent of its potential contribution to species persistence has not yet been investigated (Holyoak and Loreau, 2006). If these mechanisms create an advantage for the less abundant species, they should be classified as stabilizing, like temporal or spatial storage effects, but without invoking any differences among competitors or extrinsic environmental variation (Bertuzzo et al., 2011).

106

Here, we test the segregation hypothesis by thoroughly investigating how local dispersal and local non-specific negative density dependence impact coexistence of equivalent competitors in model communities. The model under examination is a point process in continuous space where individuals disperse offspring according to a dispersal kernel and have a rate of mortality dependent on 
of spatial patterns consistent with mapped species distribution of large tropical forest plots (Detto and Muller-Landau, 2013). We show how the spatial structure in these models develops such that there are systematic differences with focal species abundance in neighborhood densities of conspecifics and heterospecifics, and how this in turn translates to stabilization as evidenced by negative densitydependence (i.e., a rare species advantage). We explore the full parameter space which differentiates our model from the classic analytically tractable neutral model - i.e., varying dispersal distance, interaction strength and interaction range - and show how these parameters affect time to extinction in the 2-species closed system(Gandhi et al., 1998) and species diversity in the multi-species open system, two classic cases investigated in neutral theory (Beres, 2005). We also examine the effects of local community size and metacommunity species diversity, and evaluate the robustness of our results to the zero-sum assumption. This study complements the rich existing literature on spatially explicit ecological models, constituting an intermediate and necessary step towards fully understanding how niche mechanisms function in communities where individuals have limited mobility.

\subsection{Model description and simulation methods}

We first consider a closed system of $N$ individuals and two ecologically equivalent species. Parents disperse offspring according to a dispersal kernel and individual survival is a linear negative function of local density weighted by an interaction kernel, as defined below. The total number of individuals is constant, meaning that for each death there is a birth (zero-sum game). There is no immigration. The system reaches an absorbing state when one of the two species goes extinct.

In the second case study, we consider a variant of the previous model in which immigration is allowed with rate $v$ from a metacommunity with $S$ species. For simplicity, the $S$ species are equally abundant in the metacommunity (hence an immigrant has equal probability of being any one of the $S$ species). As before, the system has $N$ individuals, all species are ecologically equivalent, dispersal follows a dispersal kernel, and survival is a negative function of local density of all individuals.

We simulate these cases as point processes with $N$ individuals on a square arena of area $A$, with periodic boundary conditions to minimize edge effects. Every birth event or immigration is paired with a death event such that the total number of individuals is maintained constant. For every death event, an individual is chosen to die with a probability that depends on its neighbor density. Specifically, for each individual $j$, time to death is drawn from a random exponential distribution with rate

$$
\mu_{j}=1+\alpha \sum_{k \neq j} K\left(d_{j k}\right)
$$

where $K$ is a quadratic kernel with finite range $L_{K}$; and $d_{j k}$ is the distance between the focal individual $j$ and the neighbor $k$; then, the individual with the shortest death time is removed. For birth, a parent, chosen at random disperses an offspring according to a Gaussian kernel with parameter $L_{D}$. In the open 
system, the birth is an immigrant placed at random with probability $v$, and the offspring of a parent as before with probability 1-v. A total of $N$ deaths and $N$ births constitutes one generation time.

Initially, all $N$ individuals are placed at random. Then the models are run for an initial spin-up period to allow spatial structure to form and stabilize (initial transient time). For the closed system, individuals are initially randomly marked as species 1 or species 2 in a fixed proportion (initial state). Then, during the spin-up, each individual that dies is replaced with an individual of the same species to maintain the number of individuals per species constant. The spin-up is ended after spatial structures are fully formed (usually 5-10 generation times). For the open system, the identity of an individual is randomly chosen from a pool of $S$ species, and the rules for birth, death and immigration during the spin-up are the same as those employed thereafter. The spin-up period is continued until after the number of species reaches an equilibrium at which stochastic extinction is balanced by immigration and spatial statistics are no longer changing directionally. In practice, we used a spin-up time of 200 generation times for the open system (see supplementary material for a theoretical demonstration that this spin-up time is more than adequate for our parameter values).

\subsection{The parameter space explored}

Only three basic parameters make the difference between this spatial model and the classic spatially implicit case of Hubbell (2001): interaction strength, $\alpha$, which describes the negative influence of neighbor density on the focal individual (excluding self-interaction); dispersal distance, $L_{D^{\prime}}$, defined as the standard deviation of a Gaussian kernel; and interaction range, $L_{K}$, which is the width of a quadratic kernel representing a weighted function of distances among two interacting individuals (to make comparisons between kernels more meaningful, interaction range $L_{K}$ is multiplied by $\sqrt{6}$ to obtain an equivalent Gaussian smoothing, as in Detto and Muller-Landau (2013)). The mean density of individuals is $N / A$, which we set equal to 1 in all our simulations.

There are four asymptotic cases under which there are no spatial effects on mortality and fecundity rates: i) $L_{D} \rightarrow \infty$, ii) $L_{K} \rightarrow 0$, iii) $L_{K} \rightarrow \infty$ and iv) $\alpha \rightarrow 0$. In these cases, all species of all abundances have the same mean per capita growth rate and the mean time to extinction and relative species abundances should converge to those of the nonspatial cases.

We varied $L_{D}$ between $1 / 2$ and 2 , the distances which are half and twice as long, respectively, as the mean spacing between individuals in our simulations (suppl. Fig. 1). $L_{D}=2$ was sufficiently large to approach the first asymptotic case. Because, as shown later, this model is less sensitive to $L_{K}$, this parameter needed to be varied on a larger scale (between 1/32 and 8 ) in order to approach the asymptotic limits ii and iii. The parameter $\alpha$ also has a natural upper limit, because when $\alpha N>>(E[K(d)])^{-1}$, the mortality probability of each individual depends strongly on neighborhood density, but becomes insensitive to $\alpha$. We quantified time to extinction in the closed system for all 
180 combinations of $L_{D}=\frac{1}{2} 2^{(0,1, \ldots, 24) / 8}, L_{K}=\frac{1}{32} 2^{(0,1, \ldots, 48) / 6}$ and $\alpha=\left(\frac{1}{4}, \frac{1}{2}, 1,2\right)$. We quantified species diversity in

181 the open system for the same values of $L_{D}$ and $L_{K^{\prime}}$, together with $\alpha \in(1,2,4,8)$. Each parameter 182 combination was replicated 250 times.

Time to extinction and species diversity both increase with increasing community size, $N$, while the spatial effects we explore are qualitatively similar regardless of community size (with the exception of communities so small that spatial patterns cannot develop). Here, we used $N=50$ in our investigation of the effects of spatial parameter combinations on time to extinction in the closed system, and $N=200$ in our investigation of species diversity in the open system. Time to extinction in the closed community without spatial effects depend on community size and initial state $i$ as

$T_{i}=\sum_{j=1}^{i} \frac{N-i}{N-j}+\sum_{j=i+1}^{N-1} \frac{i}{j}$

(Watterson, 1961), motivating the smaller community size in our simulations of this case. For the open community, we further evaluated the effects of local community size $N$, varying it between 30 and 10000. In order to make comparison among simulations with different community size easier, the area $A$ was set equal to $N$ in all cases.

The open community has two additional parameters not present in the closed community case: the metacommunity species richness, $S$, and the immigration probability, $v$. Because these are not spatial parameters we do not investigate them systematically and we employ $S=200$ and $v=0.1$ in our comprehensive investigation of spatial parameter effects on diversity. We specifically explore how $S$ impacts species abundance distributions and spatial effects on diversity by comparing $S=200$ and $S=20$ for a model $N=1600$ and $v=0.01$. We explore the effect of system size, $N$, under small and large metacommunity species richness ( $S=100$ and $S=1000)$.

\subsection{Quantifying spatial effects in the simulations}

We quantified spatial structure and its variation with local abundance in simulations using Ripley's K (Ripley, 1976). In particular, we calculated the average density of conspecific neighbors and of heterospecific neighbors within distance $2 L_{D}$ from focal individuals. We computed Ripley's K for every species after the burn-in, at the end of each iteration (after birth and death events are simulated) and recorded this along with information on the species' abundance. We then averaged statistics by abundance to investigate systematic variation in spatial structure with focal species abundance. We investigated three spatial cases and the nonspatial case of the closed system to illustrate how rare species benefit from reduced crowding in the spatial models. 
We quantified variation in population growth rates with abundance in the same cases of the closed system, as well as in example cases of the open system, using the basic reproductive number, $R_{0}$, the average number of offspring produced over the lifetime of an individual. The basic reproductive number is defined as fecundity, $\lambda$, divided by mortality, $\mu$. In our model, per capita fecundity rates $\lambda$ are equal for all individuals and total abundance is constant, hence, at each realization $k, N \lambda_{k}=\sum_{j} \mu_{j k}$, where $\mu_{j k}$ is the mortality rate of the $j$ individual in the $k^{\text {th }}$ realization. Thus, the mean basic reproductive number of a species with $i$ individuals, $R_{0 i}$, can be computed as the ratio between the mortality rate of the whole community and the mortality rate of a species with $i$ individuals:

$R_{0 i}=\frac{E\left[\mu_{j} \mid s_{i}>0\right]}{E\left[\mu_{j} \mid n_{j}=i\right]}$

where $n_{j}$ is the number of individuals having the same species identity as individual $j$. Let $n_{j k}$ denote the abundance of the species of the $j^{\text {th }}$ individual in the $k^{\text {th }}$ realization, and $s_{i k}$ the number of species with $i$ individuals. Then $R_{0 i}$ is estimated as

$\hat{R}_{0 i}=\frac{\frac{\sum_{j, k} \mu_{j k}}{\sum_{k} N}}{\frac{\sum_{j \in n_{j k}=i, k} \mu_{j k}}{\sum_{k} i s_{i k}}}=\frac{\sum_{k} i s_{i k}}{k N} \frac{\sum_{j, k} \mu_{j k}}{\sum_{j \in n_{j k}=i, k} \mu_{j k}}, k \in s_{i k}>0$

The summations are carried out for all realizations $k$ where at least one species with $i$ individuals is present. It is easy to show that the integration of $1 / R_{0 i}$ over the whole community gives one, since the total population is constant. If there are no interactions ( $\alpha=0$ and/or $L_{K}=0$ ) or interactions are global $\left(L_{K}=\infty\right), \mu_{j}$ is constant for all $j$ and $R_{0 i}=1$ by definition. More generally if $E\left[\mu_{j} \mid n_{j}=i\right]=E\left[\mu_{j}\right]$, $R_{0 i}=1$.

We measured the impact of spatial effects on time to extinction in the closed system by quantifying the mean time to extinction (after the burn-in period) in 250 replicate simulations when starting with equal numbers of individuals. For the nonspatial case, the time to extinction can be calculated analytically (Appendix A).

In the open system, we measured impacts on relative species abundance distribution and species diversity. We quantified diversity using the Hill number or number of equivalent species (Hill, 1973), with $q=1$, computed as $\prod_{i=1}^{N}(i / N)^{-S_{i} i / N}$. We computed abundance distributions and species diversity as ensembles from the species abundances sampled every 5 generations for 250 generations. In the nonspatial case, the abundance distribution and thus the diversity can be obtained analytically (Appendix B). 
We investigate the invader advantage by comparing the mortality rate of a new immigrant with the mortality rate of singletons already present in the local communities, for several parameters combinations.

\subsection{The emergent dependence of mortality rates on conspecific abundance}

In our model, mortality is dependent on the local densities of heterospecifics and conspecifics, with equal effects in all species, while fecundity is density-independent (equal for all individuals). As we will show, the mortality rate $\mu$ varies systematically with the number of conspecifics $i$ when dispersal is local. (The opposite case, where fecundity is density-dependent, can be studied in a similar manner and has similar behavior.)

The mortality rate of a focal species $\mathrm{X}$ with $i$ individuals can be divided into three components: a density-independent component (fixed arbitrarily at 1), a component representing effects of conspecific interactions (individuals of the focal species X), and a component representing effects of heterospecific interactions (individuals of all species other than X). This can be expressed as

$\mu_{\mathrm{X}}=1+\alpha \frac{1}{i} \sum_{j \in \mathrm{X}} \sum_{k \neq j, k \in \mathrm{X}} K\left(d_{j k}\right)+\alpha \frac{1}{i} \sum_{j \in \mathrm{X}} \sum_{k \notin \mathrm{X}} K\left(d_{j k}\right)$

where $\alpha$ is the strength of the interaction, $d_{j k}$ is the distance between the focal individual $k$ and the neighbor $j$, and $K$ is an interaction kernel with finite range $L_{k}$. The condition $k \neq j$ is to exclude selfinteraction. Using conditional expectations, (5) can be rewritten as

$\mu_{\mathrm{X}}=1+\alpha(i-1) E\left[K\left(d_{j k}\right) \mid k \neq j, I D_{j}=I D_{k}=I D_{\mathrm{X}}\right]+\alpha(N-i) E\left[K\left(d_{j k}\right) \mid I D_{j}=I D_{\mathrm{X}}, I D_{k} \neq I D_{\mathrm{X}}\right](6)$

where $I D$ denotes species identity. In the presence of intraspecific aggregation and interspecific segregation it is reasonable to assume the following inequality:

$E\left[K\left(d_{j k}\right) \mid k \neq j, I D_{j}=I D_{k}\right]>E\left[K\left(d_{j k}\right) \mid I D_{j} \neq I D_{k}\right]$

Inequality (7) expresses the fact that pairwise distances between conspecific are on average smaller than distances between heterospecifics. In particular, for a singleton in a two-species community with $N$ individuals we have

$\mu_{1}=1+\alpha(N-1) E\left[K\left(d_{j k}\right) \mid I D_{j} \neq I D_{k}\right]$

which, in virtue of inequality (7), is smaller than

$\mu_{N-1}=1+\alpha(N-2) E\left[K\left(d_{j k}\right) \mid j \neq k, I D_{j}=I D_{k}\right]+\alpha E\left[K\left(d_{j k}\right) \mid I D_{j} \neq I D_{k}\right]$ 
Although approximate solutions have been proposed for spatially explicit Lotka-Volterra models (e.g. Adler and Muller-Landau, 2005; Bolker and Pacala, 1997; Neuhauser and Pacala, 1999), a closed expression for the conditional expectations is not known. Furthermore, instantaneous rates deviate from expected values for finite population size, since they depend on the exact locations of the individuals at a specific point in time. To understand how mean time to extinction and diversity change as function of model parameters, we thus rely on computer simulations.

\section{RESULTS}

The net impacts of conspecific aggregation and heterospecific segregation in the spatial model vary systematically with the focal species abundance in the closed two-species community (Figure 1A,B). Rare species suffer less from conspecific aggregation and benefit more from heterospecific segregation than do common species, and thus experience higher basic reproductive numbers in the spatial model, but not in the nonspatial model (Figure $1 C$ ). The two species have equal probability to gain or lose an individual when they have equal relative abundance (0.5). When a species becomes rare, it gains a demographic advantage that provides it with a chance to recover, and that advantage increases with the strength of local interactions (Figure 1C).

Consistent with this rare species advantage, mean time to extinction in the spatial model of a closed two-species community was substantially longer than for the corresponding nonspatial model (Figure 2). For the nonspatial case, mean time to extinction in simulations agreed well with the analytical solution obtained for birth and death rates independent of species relative abundance (Eq.2), with time to extinction depending only on system size and initial state (Figure 2, red points vs. red line). The spatial case shows a similar dependence on initial state, but mean time to extinction is significantly longer whenever parameters are such that substantial spatial aggregation and segregation develops (e.g., Figure 2, blue points). All spatial parameters influenced the degree to which extinction times were extended in the spatial model (Figure 3). Extinction times were prolonged by shorter dispersal distances, more intense negative density-dependence (higher $\alpha$ ), and interaction ranges between $1 / 2$ and the dispersal distance.

The multi-species system with immigration exhibits qualitatively the same pattern of rare species advantage in basic reproductive number (Figure 4, top). The basic reproductive number declines with increasing relative abundance, crossing one at a relative abundance equal to the inverse of expected number of species for those parameters. In this system, rare species can arise not only from common species that have declined, but also as immigrants. Immigrant seeds have a greater probability to land in low-density areas than residents' seeds. As a result, invader reproductive numbers are elevated above one and increase with interaction strength (Figure 4, bottom). The advantage of the invader is also increased under conditions of greater aggregation such as those that develop under shorter dispersal (Figure 4, bottom). When we remove the zero-sum constraint and allow the density of individuals to be determined by the balance of births and deaths, the pattern of basic reproductive number declining with increasing abundance remains qualitatively unchanged (Appendix C). 
Species diversity in the spatial multi-species model with immigration was higher than in the parallel nonspatial model (Figure 5). Diversity was higher for shorter dispersal distances, higher interaction strength, and interaction ranges between $1 / 2$ and the dispersal distance (Figure 5). These patterns of variation are qualitatively the same as those found for extinction times in the closed system (compare Figure 3 and Figure 5). Abundance distributions also differed. The spatial model exhibits a reduction in dominance (fewer very abundant species) and an increase in the number of species of intermediate abundance relative to the nonspatial model (Figure 6). The effect on species abundance distributions depended also on the size of the metacommunity: for species-poor metacommunities, but not species-rich ones, spatially localized interactions greatly reduced rarity.

For a given immigration rate, the diversity enhancement due to local interactions becomes weaker as the size of the local community increases (Figure 7). For local communities that are relatively small compared to the regional species pool, the increased diversity is independent of regional pools. For relatively larger local communities $(N \gg>S)$, the differences between the spatial and nonspatial cases vanish as the limit imposed by the regional pool is reached.

ur results clearly show that the segregation effect (Pacala and Levin, 1997; Pacala, 1997) stabilizes coexistence of equivalent competitors in continuous space models with spatially localized dispersal and competitive interactions, even when competitive effects and responses are entirely generalized (species identity of individuals does not matter). Such generalized interactions can arise if the species compete for the same resources or share a common enemy (apparent competition). The basic reproductive number declines with increasing abundance in the spatial model unequivocally demonstrates that the aggregation-segregation mechanism qualifies as stabilizing (Peter Chesson, 2000). The results are robust to the zero-sum assumption and are applicable to models where total number of individuals change with time, e.g. logistic processes (see Appendix $C$ for an example).

Maximum stabilization is achieved with high dispersal limitation and with strong interactions at comparable (hence short) scales. Although variation in interaction range has less influence than variation in dispersal distance, it is notable that the scales of these two factors interact. If dispersal is large compared to the simulated arena, individuals are placed at random and the neighbor densities are uncorrelated with focal individual identity. Similarly, if interaction range is large compared to the simulated arena, neighbor density equals mean field density for all individuals, independently of identity. On the other hand, if interaction range is small compared to dispersal and mean spacing among individuals, individuals do not interact with neighbors and mortality is determined by densityindependent factors. The two cases examined, the closed and open systems, are analogous, and produce fundamentally consistent conclusions. The key distinction is that in the open local community, immigrants enter randomly, i.e., as if dispersed long distances. This gives an additional advantage to immigrants that can land in unoccupied areas that are temporarily out of reach of residents, thus 
increasing the average rare species advantage. This is consistent with analytical results of Bolker and Pacala (1999) and Murrell (2010)

The effects of spatially localized interactions on diversity vary with immigration, highlighting the importance of regional richness for these types of spatial neutral interactions. For rich regional species pools, the diversity-enhancing effect of local interactions is achieved by a reduction in relative abundance of the dominant species. These individuals are redistributed into lower abundance classes, naturally increasing the species of intermediate abundance. The number of rare species, in contrast, remains relatively unchanged. This is because the fast rate of immigrants from the rich regional pool is compensated by the greater advantage of rare species due to spatial dynamics. In contrast, for communities embedded in poor regional species pools, there is a stronger reduction in the number of rare species because the advantage of being rare is not compensated by a continuous immigration of new species. Analogously, species-area curves saturate when the community size becomes large enough to include the whole regional species pool, with the consequence of minimizing locally stabilizing effects in general, including the spatial effects addressed here.

\section{Understanding stabilization due to the aggregation-segregation effect}

The stabilization observed in the continuous space models investigated here is due to the combination of conspecific aggregation and interspecific segregation, which are not completely compensatory in continuous space models (Bolker et al., 1999). The stabilization emerges in a dynamical way through continuous formation and dissipation of spatial structures (Detto and Muller Landau, in press). Formation of spatial structures occurs where reproduction is successful, but dispersal is limited, leading inevitably to the formation of dense clusters.

Dissipation of spatial structures occurs because negatively density-dependent mortality erodes dense clusters. In these models, individual species abundances fluctuate like a biased random walk, bounded from 0 to $N$. Conspecific neighborhoods around survivors of a species that has experienced negative drift are relatively less dense (due to dissipation of spatial structures). Because species are segregated, the net effect is that less abundant species are subjected to relatively less local competition. In contrast, individuals of a species that has experienced positive drift will have on average denser neighborhoods (due to the formation of spatial structures), with a net effect of increased local competition. Essentially, when dispersal and competition are spatially localized, spatial patterns and abundance change over time in such a way that spatial statistics covary systematically with abundance (figure 1). This mechanism can also be thought of as generating a kind of spatial refugia, i.e., locations where relict populations of once more widespread species remain isolated, favoring persistence of these species after their extinction in surrounding areas. This phenomenon cannot arise in lattice models in which all cells are always filled, as the total densities of neighbors remains constant regardless of abundance in such models.

In the past, it has been argued that the effect of local dispersal and interactions in elevating diversity (e.g., Chave et al. 2002) is never a stabilizing mechanism, that it merely slows drift to extinction rather 
than really creating stable coexistence (Peter Chesson, 2000). However, in finite, stochastic communities without immigration, virtually all niche mechanisms "merely" slow drift to extinction. The crucial distinction of a stabilizing mechanism is that it elevates a species' per capita growth rate when it becomes rare, tending to aid recovery (stochastic boundedness), and thus, a stabilizing effect is evident here.

385

386

387

388

389

390

391

392

393

394

395

Chesson (2000b) distinguished three main categories of stabilizing mechanisms: fluctuationindependent mechanisms, those involving nonlinearity of competition, and storage effects in space or time. The mechanism studied here is a fluctuation-dependent mechanism, because it depends on spatiotemporal variability in population density which covaries with growth rates: the differences in densities among patches disproportionally affect mortality in the patches (similar results would be obtained if NDD instead affected fecundity or establishment). However, there are two important differences between traditionally defined fluctuation mechanisms (e.g. the storage effect) and the spatial segregation effects seen here. First, fluctuations in population density are generated not by exogenous spatial or temporal environmental variability, but by endogenous stochastic demography. Second, there are no species-specific responses to the environment; all species are equivalent in their responses to neighbors.

\section{Relationship to previous studies}

Previous studies of continuous spatial models have focused on asymmetric cases in which conspecifics exert more negative neighborhood effects than heterospecifics, generally inspired by models of specialized natural enemies (e.g. Adler and Muller-Landau, 2005; Sedio and Ostling, 2013). Some of these papers nonetheless provide analytical or simulation results on models with nonspecific interactions as well.

Bolker and Pacala (1999) analyzed continuous spatially explicit Lotaka-Volterra models, the same general type of models used in Murrell and Law (2003) and in our study, except that in their case density-dependence affected establishment rather than mortality, and the shape of dispersal and competition kernels are qualitatively different. They analytically derived invasion criteria for the spatial model as a function of spatial and nonspatial parameters. These criteria have the general form that the invasion rate is equal to the nonspatial invasion rate (zero for equivalent competitors) plus a term for the resident's aggregation, minus a term related to the invader's aggregation, and plus a term for resident-invader segregation (Eq. 6 in Bolker and Pacala 1999). A randomly placed invader initially has zero conspecific aggregation and zero heterospecific segregation, and thus the spatial invasion growth rate reduces simply to a positive term for the aggregation of the resident in the case of equivalent competitors. Similar results were obtained by Murrell (2010) for the case of invasion into a resident population with spatial structure. These analytical results are consistent with our finding of stabilizing effects of the aggregation-segregation mechanism in continuous spatial models. In contrast, the analytical results of Murrell and Law (2003) suggest that asymmetries in interaction ranges between conspecifics and heterospecifics NDD are a necessary condition for space alone to mediate coexistence. Our results are obtained with a symmetric competition kernel in which conspecific and heterospecific 
interaction distances and strengths are equivalent, and thus fall on the boundary between stably coexisting and bistable states, according to the analysis of Murrell and Law (2003).

Previous simulation studies of continuous spatial models also present some relevant results. Sedio and Ostling (2013) focus on how the degree of specialization in natural enemies influences species richness, and present models with completely generalist natural enemies (i.e., nonspecific neighborhood interactions) for comparison. Their results show that under multiple parameter combinations, models with generalist natural enemies support higher diversity than neutral models lacking spatial processes (their Fig 3a, S1a). Adler and Muller-Landau (2005) focus on how spatial scales of dispersal and conspecific interactions affect species richness, but also present results for different scales of heterospecific interactions. Simulations with short seed dispersal distances and short heterospecific interaction distances have much higher species richness than those with longer dispersal and interaction distances (their Table 2).

\section{Directions for future research}

Future research could usefully expand on the present work through extension of the spatially explicit model to the metacommunity, extension to more complex interactions (Perc et al., 2013), and analytical results for these models. The results presents above are restricted to unstable coexistence problems of neutral communities in continuous space. Different model formulations may not generate stabilizing effects and in models with species differences some spatial structures may even reduce the parameters space where stable coexistence occurs (Neuhauser and Pacala, 1999) or accelerate exclusions (Bolker et al., 1999). For example we have already noted that a lattice neutral model without empty space does not produce local density fluctuations, thereby excluding the possibility of fluctuation-dependent stabilizing mechanisms.

The current model explicitly represents spatial interactions within the local community, but relies on a spatially implicit approach to immigration from the metacommunity. A complete spatial explicit modelling of metacommunity and local community should represent immigration as dispersal from a limited area in proximity to the local community. This would reduce lead to nonrandom sampling of the metacommunity (Hubbell, 2001) and nonrandom placement of immigrants. Nonrandom sampling of the metacommunity would cause the local community $t$ a smaller metacommunity. Nonrandom placement of immigrants would mean that immigrants are likely to have conspecifics nearby (even if just over the "border" of the local community), reducing the invader advantage (Chisholm and Lichstein, 2009; Ostling, 2012). Both factors would lead to a reduction in species richness. Essentially, this approach requires explicitly simulating the entire metacommunity itself, a task that obviously depends greatly upon available computing power.

It would also be useful to extend this model to more complex interactions involving multiple trophic levels, such as host-parasitoid interactions. This would elucidate the role of spatial effects in competition among species with a common (generalist) enemy (Bonsall and Hassell, 2000; King and Hastings, 2003). The crucial difference from neighborhood competition models such as the ones 
employed here is that natural enemies do not respond instantaneously, and thus dynamics exhibit lags that affect the rates at which spatial structures form and dissipate (Detto and Muller-Landau, in press).

Finally, there is a strong demand for analytical treatment of this problem (Harte et al., 2008; Holyoak and Loreau, 2006). One possibility is to use spatial moment equations to express per capita population rates as sum of nonspatial and spatial components, where the latter are dependent on the spatial covariance and the interaction kernel (Bolker and Pacala 1999). However, in moment equations, as well as in reaction-diffusion equations, some properties of the discreetness of the system cannot yet be easily incorporated. For examples, even if the spatial moments are known analytically and the expected mortality and fecundity rates are resolved, the finite state Markov chain cannot be implemented.

While theoretical investigation of the deviation from neutral theory in spatially explicit models has focused on the importance of niche mechanisms, relatively less attention has been given to neutral spatial mechanisms. Here, we demonstrated that, in continuous space models, spatial interactions, even when completely non-specific, can modify classic results for communities driven by ecological drift, because they introduce a stabilizing mechanism. The aggregation-segregation effect described here is pervasive across several orders of magnitude in local community size, suggesting that many different natural ecosystems may be influenced by such mechanism. Because neutral local interactions may have significant effects on the dynamics of the system, these effects needs to be parsed out first whenever spatially explicit models are investigated to quantify stabilizing contributions of particular niche mechanisms.

\section{Acknowledgements}

We thank Peter Chesson and several anonymous reviewers for helpful comments on an earlier version of this manuscript. This work was supported by Smithsonian Institution Global Earth Observatory (SIGEO).

\section{References}

Adler, F.R., Muller-Landau, H.C., 2005. When do localized natural enemies increase species richness? Ecol. Lett. 8, 438-447. doi:10.1111/j.1461-0248.2005.00741.x

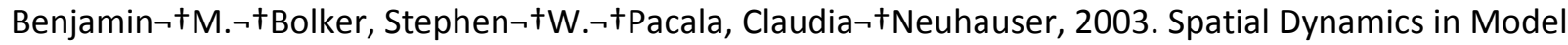
Plant Communities: What Do We Really Know? Am. Nat. 162, 135-148. doi:10.1086/376575

Beres, K., 2005. Rotifers and Hubbell's unified neutral theory of biodiversity and biogeography. Nat. Resour. Model. 18, 363-376. doi:10.1111/j.1939-7445.2005.tb00163.x

Bertuzzo, E., Suweis, S., Mari, L., Maritan, A., Rodríguez-iturbe, I., Rinaldo, A., 2011. Spatial effects on 
species persistence and implications for biodiversity. Proc. Natl. Acad. Sci. U. S. A. 108, 4346-4351. doi:10.1073/pnas.1017274108

Bolker, B., Pacala, S., 1997. Using Moment Equations to Understand Stochastically Driven Spatial Pattern Formation in Ecological Systems. Theor. Popul. Biol. 52, 179-97.

Bolker, B.M.B., Pacala, S.W.S., The, S., Naturalist, A., June, N., Bolker, B.M.B., Pacala, S.W.S., 1999. Spatial moment equations for plant competition: understanding spatial strategies and the advantages of short dispersal. Am. Nat. 153, 575-602.

Bonsall, M.B., Hassell, M.P., 2000. The effects of metapopulation structure on indirect interactions in host-parasitoid assemblages. Proc. Biol. Sci. 267, 2207-2212. doi:10.1098/rspb.2000.1270

Britton, N., 1986. Reaction-diffusion equations and Their application to Biology. Academic Press, New York.

Chave, J., Muller-Landau, H.C., Levin, S.A., 2002. Comparing classical community models: theoretical consequences for patterns of diversity. Am. Nat. 159, 1-23. doi:10.1086/324112

Chesson, P., 2000. General theory of competitive coexistence in spatially-varying environments. Theor. Popul. Biol. 58, 211-37. doi:10.1006/tpbi.2000.1486

Chesson, P., 2000. Mechanisms Ofmaintenance of Species Diversity. Annu. Rev. Ecol. Syst. 31, 343-66.

Chesson, P., Neuhauser, C., 2002. Intraspecific aggregation and species coexistence - Comment from Chesson and Neuhauser. Trends Ecol. Evol. 17, 210. doi:10.1016/s0169-5347(02)02504-1

Chisholm, R.A., Lichstein, J.W., 2009. Linking dispersal, immigration and scale in the neutral theory of biodiversity. Ecol. Lett. 12, 1385-1393. doi:10.1111/j.1461-0248.2009.01389.x

Detto, M., Muller-Lanau, H., 2016. Rates of formation and dissipation of clumping reveal lagged responses in tropical tree populations. Ecology. doi:10.1890/15-1505.1

Detto, M., Muller-Landau, H.C., 2013. Fitting ecological process models to spatial patterns using scalewise variances and moment equations. Am. Nat. 181, E68-82. doi:10.1086/669678

Dunning, J.B., Stewart, D.J., Danielson, B.J., Noon, B.R., Root, T.L., Stevens, E.E., 1995. Spatially Explicit Population Models : Current Forms and Future Uses 1. Ecol. Appl. 5, 3-11.

Durrett, R., Levin, S.A., 1994. The Importance of Being Discrete (and Spatial). Theor. Popul. Biol. 46, 363394. doi:10.1006/tpbi.1994.1032

Etienne, R.S., Rosindell, J., 2011. The spatial limitations of current neutral models of biodiversity. PLoS One 6, e14717. doi:10.1371/journal.pone.0014717

Fisher, R. a, Corbet, a S., Williams, C.B., 1943. The relation between the number of species and the number of individuals in a random sample of an animal population. J. Anim. Ecol. 12, 42-58. doi:10.2307/1411

Gandhi, A., Levin, S., Orszag, S., 1998. "Critical slowing down" in time-to-extinction: an example of critical phenomena in ecology. J. Theor. Biol. 192, 363-76. doi:10.1006/jtbi.1998.0660

Hanski, I., 1981. Coexistence of competitiors in patchy environments with and without predation. Oikos 37, 306-312. doi:10.1377/hlthaff.2010.0023

Harte, J., Zillio, T., Conlisk, E., Smith, a B., 2008. Maximum entropy and the state-variable approach to macroecology. Ecology 89, 2700-2711. doi:10.1890/07-1369.1

Hill, M., 1973. Diversity and evenness: a unifying notation and its consequences. Ecology 54, 427-432. doi:10.2307/1934352 
Holyoak, M., Loreau, M., 2006. Reconciling empirical ecology with neutral community models. Ecology 87, 1370-1377. doi:10.1890/0012-9658(2006)87[1370:REEWNC]2.0.CO;2

Hubbell, S.P., 2001. MacArthur and Wilson's radical theory. Unified Neutral Theory Biodivers. Biogeogr. 18, 3-29. doi:10.1111/j.1939-7445.2005.tb00163.x

Hurtt, G.C., Pacala, S.W., 1995. The consequences of recruitment limitation: reconciling chance, history and competitive differences between plants. J. Theor. Biol. 176, 1-12. doi:10.1006/jtbi.1995.0170

Karlin S, T.H.M., 2010. An Introduction to Stochastic Modeling, Third Edition, 3rd ed, Star. Academic Press, San Diego.

King, A.A., Hastings, A., 2003. Spatial mechanisms for coexistence of species sharing a common natural enemy. Theor. Popul. Biol. 64, 431-438. doi:10.1016/S0040-5809(03)00100-X

Molofsky, J., Bever, J.D., Antonovics, J., Newman, T.J., 2002. Negative frequency dependence and the importance of spatial scale. Ecology 83, 21-27. doi:10.1890/00129658(2002)083[0021:NFDATI]2.0.CO;2

Murrell, D.J., 2010. When does local spatial structure hinder competitive coexistence and reverse competitive hierarchies? Ecology 91, 1605-1616. doi:10.1890/09-0832.1

Murrell, D.J., Law, R., 2003. Heteromyopia and the spatial coexistence of similar competitors. Ecol. Lett. 6, 48-59. doi:10.1046/j.1461-0248.2003.00397.x

Murrell, D.J., Purves, D.W., Law, R., 2001. Uniting pattern and process in plant ecology. Trends Ecol. Evol. 16, 529-530. doi:10.1016/S0169-5347(01)02292-3

Neuhauser, C., Pacala, S.W., 1999. An explicitly spatial version of the Lotka-Volterra model with interspecific competition. Ann. Appl. Probab. 9, 1226-1259. doi:10.1214/aoap/1029962871

O’Brien, E.E., Brown, J.S., Moll, J.D., 2007. Roots in space: a spatially explicit model for below-ground competition in plants. Proc. R. Soc. Biol. Sci. 274, 929-934. doi:10.1098/rspb.2006.0113

Ostling, A.M., 2012. Large-scale spatial synchrony and the stability of forest biodiversity revisited. J. Plant Ecol. 5, 52-63. doi:10.1093/jpe/rtr035

Pacala, S.W., 1987. Neighborhood models of plant population dynamics 3. Models with spatial heterogeneity in the physical environment. Theor. Popul. Biol. 31, 359-392. doi:10.1016/00405809(87)90012-8

Pacala, S.W., Levin, S.A., 1997. Biologically Generated Spatial Pattern and the Coexistence of Competing Species, in: Tilman, D., Kareiva, P. (Eds.), Spatial Ecology: The Role of Space in Population Dynamics and Interspecific Interactions. Princeton Univ. Press, Princeton, pp. 204-2330.

Pacala, W., 1997. Dynamics of Plant Communities, in: Crawley, M. (Ed.), Plant Ecology. Blackwell Science Ltd, Oxford, pp. 532-555. doi:10.1002/9781444313642.ch15

Perc, M., Gomez-Gardenes, J., Szolnoki, A., Floria, L.M., Moreno, Y., 2013. Evolutionary dynamics of group interactions on structured populations: a review. J. R. Soc. Interface 10, 20120997. doi:10.1098/rsif.2012.0997

Ripley, B., 1976. The Second-Order Analysis of Stationary Point Processes. J. Appl. Probab. 13, 255-266. doi:10.1111/j.1365-2672.2007.03484.x

Sedio, B.E., Ostling, A.M., 2013. How specialised must natural enemies be to facilitate coexistence among plants? Ecol. Lett. 16, 995-1003. doi:10.1111/ele.12130

Slatkin, M., 1974. Competition and Regional Coexistence. Ecology 55, 128-134. doi:10.2307/1934625 
572 Stoll, P., Prati, D., 2001. Intraspecific Aggregation Alters Competitive Interactions in Experimental Plant

573

574

575

576

577

578

579

580

581

582

583

584 Communities INTRASPECIFIC AGGREGATION ALTERS COMPETITIVE INTERACTIONS IN EXPERIMENTAL PLANT COMMUNITIES. Ecology 82, 319-327. doi:10.2307/2679862

Turnbull, L.A., Coomes, D.A., Purves, D.W., Rees, M., 2007. How spatial structure alters population and community dynamics in a natural plant community. J. Ecol. 95, 79-89. doi:10.1111/j.13652745.2006.01184.x

Watterson, G.A., 1961. Markov chains with absorbing states: A genetic example. Ann. Math. Stat. 32, 716-729.

Webb, S.D., Keeling, M.J., Boots, M., 2007. Host-parasite interactions between the local and the meanfield: How and when does spatial population structure matter? J. Theor. Biol. 249, 140-152. doi:10.1016/j.jtbi.2007.06.013 

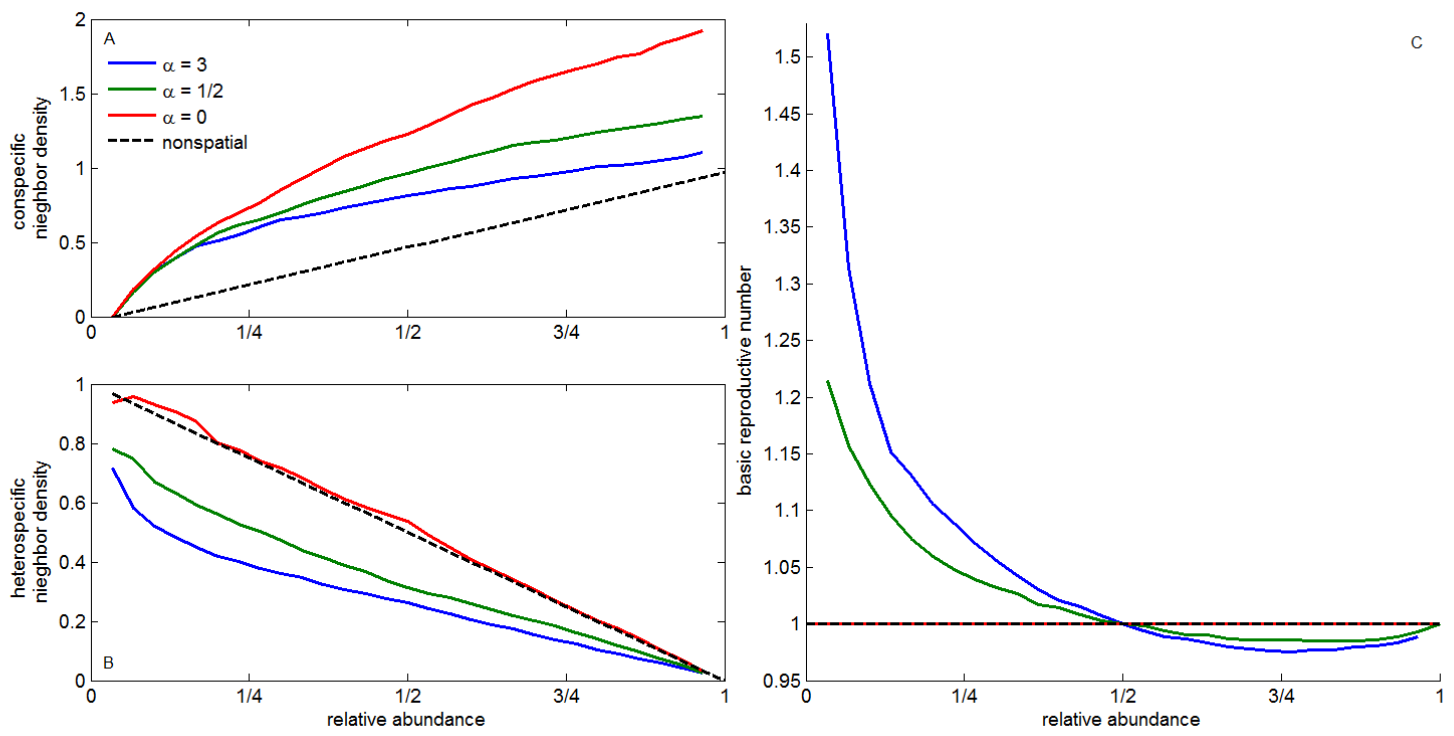

587 Figure 1. Conspecific aggregation, heterospecific segregation, and basic reproductive number vary systematically with focal species abundance in the closed two-species system under local dispersal and competition ( $N=30, L_{D}=$ $L_{K}=1 / 2$ ), compared with the nonspatial case (black dashed lines). (A) Conspecific aggregation is higher in the cases with local dispersal than in the nonspatial case, and the increase in aggregation is greater for common species than for rare species. (B) Heterospecific segregation is stronger (lower local heterospecific density) in the cases with nonzero local competition than in the nonspatial cases, with rare species benefiting more from segregation than do common ones, as they experience a greater reduction in local heterospecific density. (C) The net effects of the changes in conspecific and heterospecific neighbor density is that species benefit from lower total neighborhood density when rare, such that the basic reproductive number (average offspring produced per individual) declines with increasing relative abundance. Note that increasing the strength of local competition $(\alpha)$ reduces conspecific aggregation and increases heterospecific segregation, with a net strengthening of the spatial effect of densitydependence. Neighbor density (A, B) is measured as the density of individuals within distance 1 from focal individuals.

600 


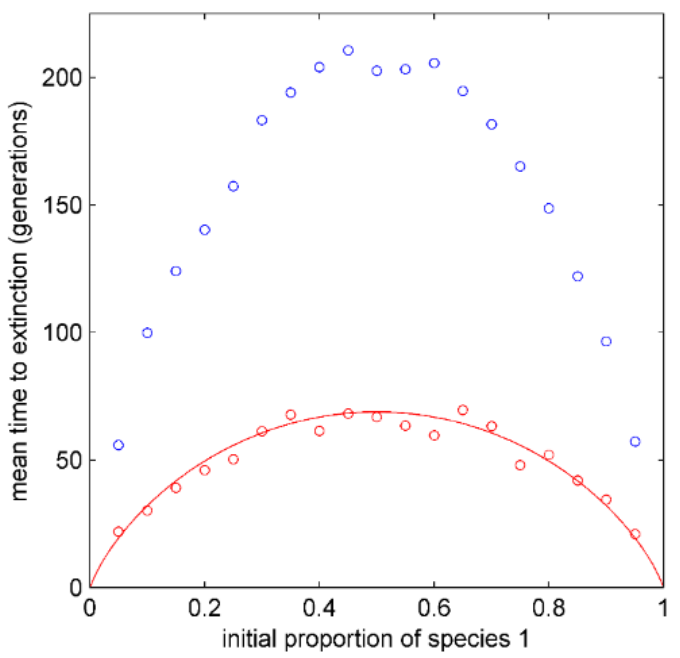

603 Figure 2. In a closed two-species community, mean time to extinction of one species is higher in a spatial model with local dispersal and local density-dependent mortality (blue circles) than in a nonspatial model (red circles; red line is the analytical solution). Species are ecologically equivalent in both models; in the spatial case, individuals have limited dispersal and local interactions (with identical effects of and on conspecifics and heterospecifics). Means were taken over 1000 simulations with a system size $N=100$. Parameters of the spatial model $L_{D}=L_{C}=1 / 4$ and $\alpha=5$. 

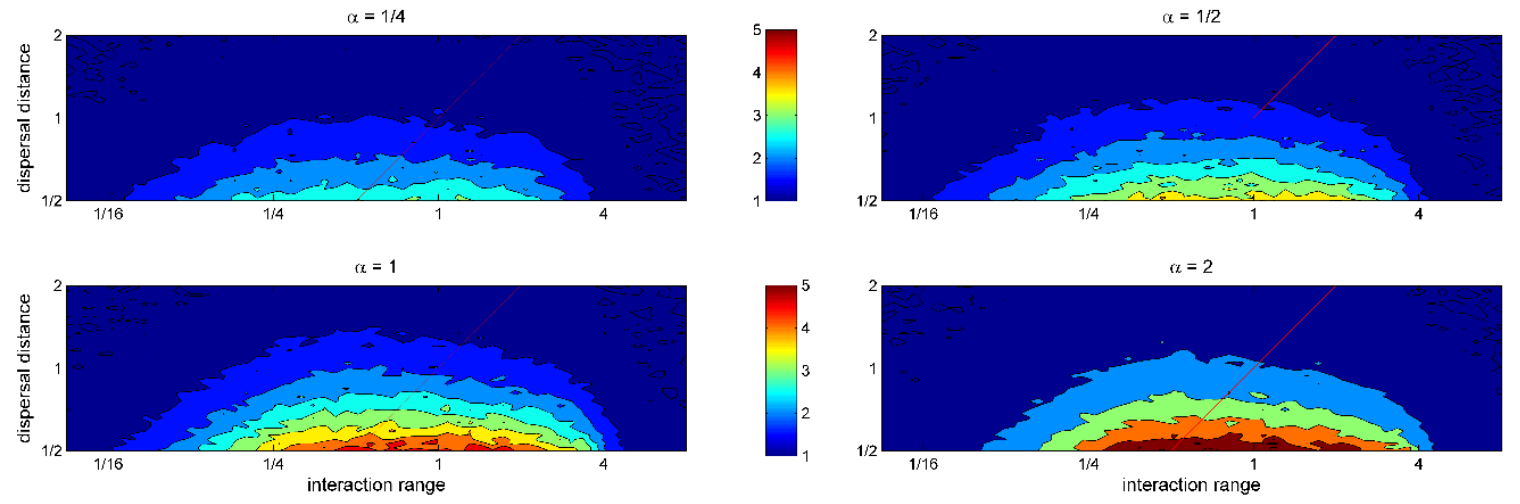

612 Figure 3. The ratio of mean time to extinction in the spatial model to that in the nonspatial case varies with

613 interaction range ( $L_{K}, \mathrm{x}$ axis), dispersal distance $\left(L_{D}, \mathrm{y}\right.$ axis), and interaction strength ( $\alpha$, panels). Extinction time in

614 the spatial model increases with shorter dispersal distance, stronger interaction strength, and interaction ranges

615 between $1 / 2$ and the dispersal distance. Means were taken over 250 simulations with system size $N=50$ and initially

616 equal abundances of both species. The diagonal line indicates the parameter combinations for which interaction

617 range equals dispersal distance. 

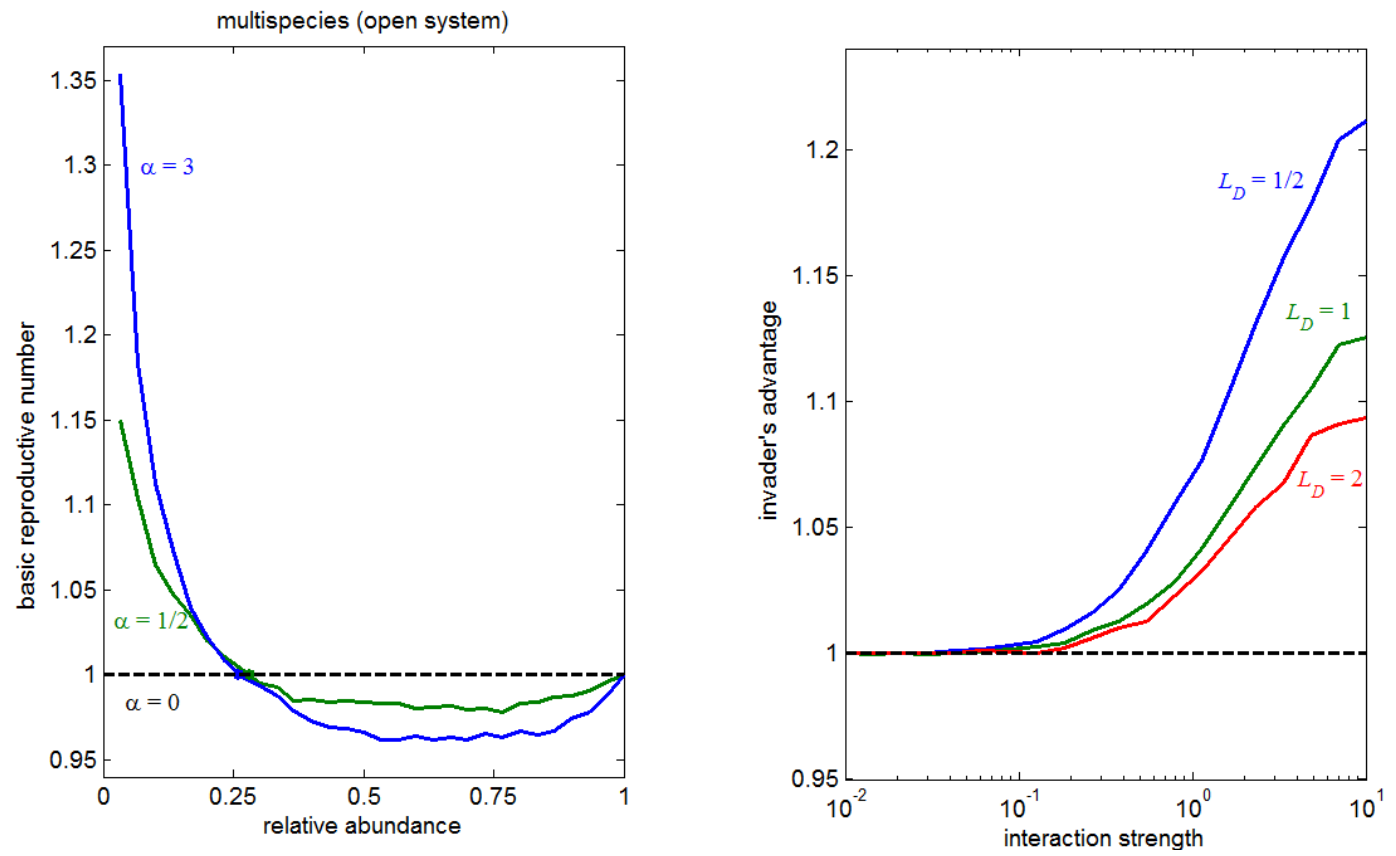

622 Figure 4. Left) The basic reproductive number $R_{0 i}$ declines as a function of relative abundance in the multispecies system with immigration, with steeper declines for higher interaction strengths $\alpha$ (lines). The dots indicate the reproductive number when all species have the same relative abundance. Reproductive numbers are computed using 300,000 realizations after burn-in time with $N=30, L_{D}=L_{K}=1 / 2, S=5, v=0.05$. Bottom) Right) Invader's advantage increases with local interaction strength, and dispersal limitation of the residents. Other parameters are $N=30, S=5, v=0.05$. Invader's advantage is defined as the ratio between average mortality rates of a singleton resident species and an immigrant before it generates an offspring. Mortality rates are computed using 300,000 realizations after burn-in time. 

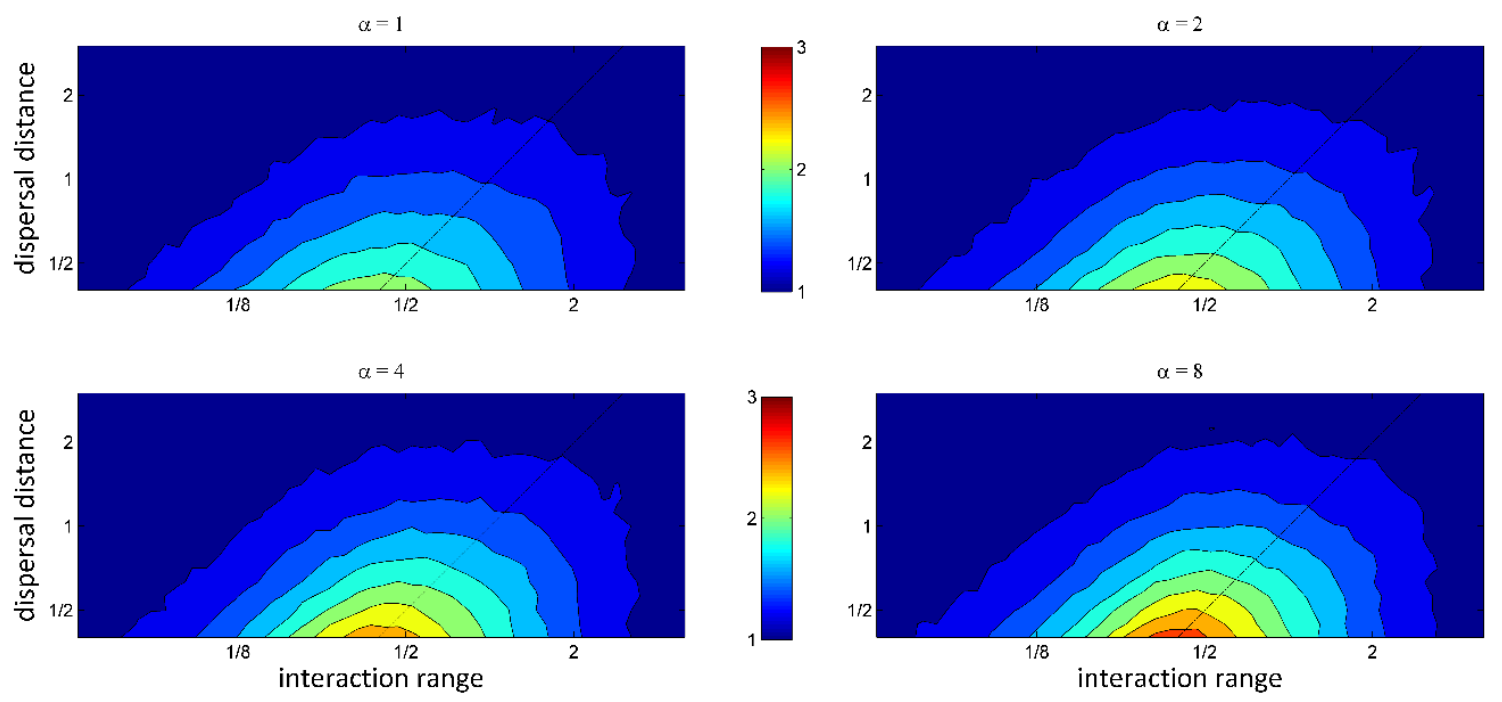

632

633 Figure 5. In the multi-species community with immigration, the ratio of species diversity in the spatial relative to 634 that in the nonspatial model varies with interaction range $\left(L_{K}, \mathrm{x}\right.$ axis), dispersal distance $\left(L_{D}, \mathrm{y}\right.$ axis), and

635 interaction strength ( $\alpha$, panels). Diversity in the spatial model increases with shorter dispersal distance, stronger 636 interaction strength, and interaction ranges between $1 / 2$ and dispersal distances. Diversity is calculated as the Hill 637 number of equivalent species (see Methods). Means were taken over 250 simulations with system size $N=200$, metacommunity size $S=200$, and immigration rate $v=0.1$. The diagonal line indicates the parameter combinations for which interaction range equals dispersal distance.

640

641 

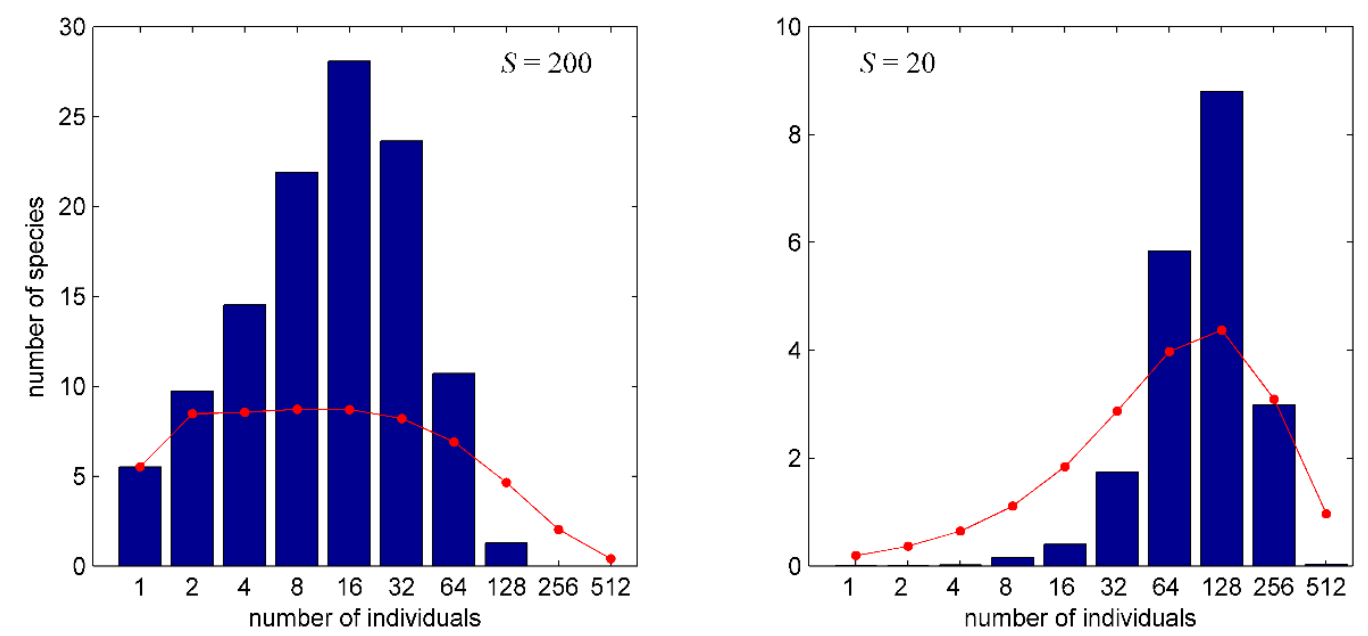

644 Figure 6. Species abundance distributions for simulated communities with local dispersal and interactions (blue 645 bars) deviate systematically from analytical solutions for nonspatial communities (red dots and lines, Eq. 8), with 646 different deviations for the cases of large (left) and small (right) metacommunities. Abundance distributions are 647 presented as numbers of species in doubling classes of abundance (aka Preston plot). Model parameters: $N=1600$, $648 L_{D}=L_{C}=3 / 4, v=0.01, \alpha=10$. When $\mathrm{S}=200$, the Hill number of equivalent species is $\mathrm{X}$ for the spatial case 649 compared with $X$ for the nonspatial case; at $S=20$, the Hill numbers are $X$ and $X$. 


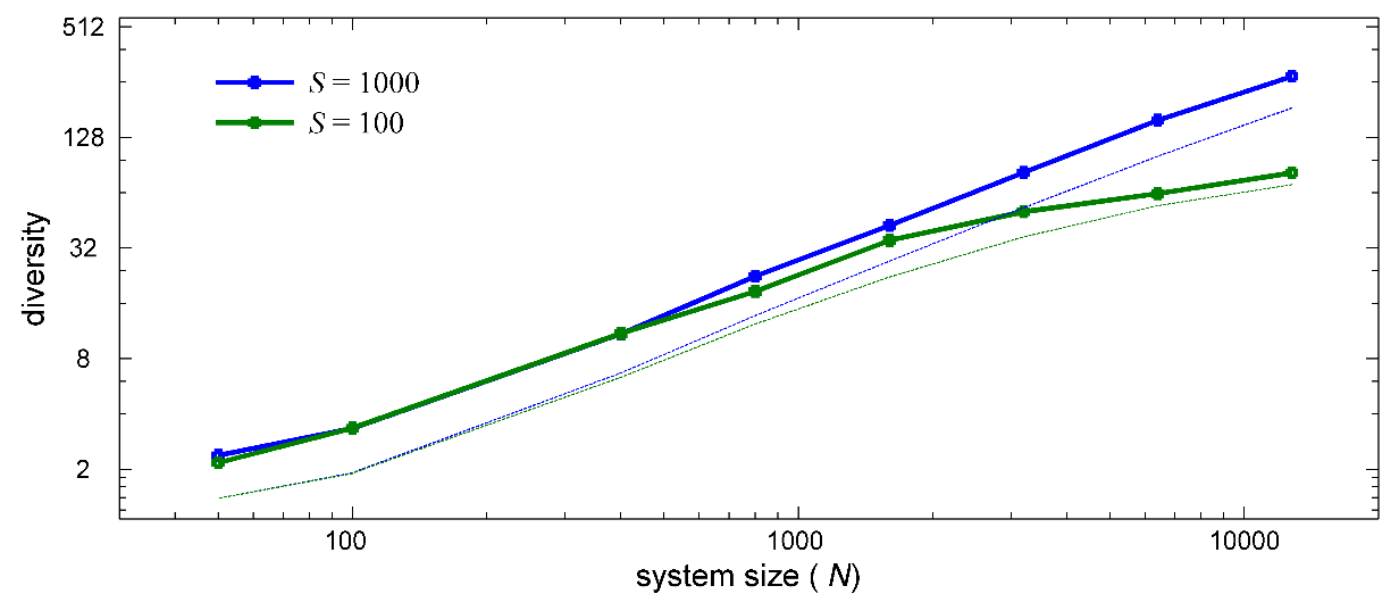

652 Figure 7. Effect of system size (number of individuals in the local community), on the difference in diversity 653 between the nonspatial (thin dashed lines) and spatial (filled circles and thick solid lines) cases under relatively 654 small (green) or large (blue) regional species pools. For the nonspatial model, the analytical solutions are shown; 655 the spatial cases are simulation results (points, means over 250 simulations). Parameter values: $L_{D}=L_{C}=1, v=$ $6560.01, \alpha=10$. 